\title{
Final Reflections
}

Throughout this book we have tried to provide a broad view of some of the different levels of analysis that are involved in the study of pottery production in archaeology and, especially, in the study of pottery pastes and fabrics using archaeometric methodologies.

In this sense, rather than focusing our efforts on describing in detail the wide range of techniques or methodologies currently used in the characterisation of ceramic materials, we have chosen to consider in detail some of the main theoretical frameworks from which ceramic technology is usually interpreted. This has involved dealing with the main concepts and viewpoints currently used in archaeological research as well as highlighting their possibilities and constraints in order to promote an archaeological praxis that may allow developing more complex discourses about past societies which also possess greater internal consistency.

In this sense a wide overview of certain aspects of the pottery analysis was presented, considering the main perspectives that can be applied when undertaking the study of pottery technology and the analysis of pastes and fabrics. Thus, the ultimate goal of this book is to encourage reflection, especially by those researchers who face the analysis of ceramics for the first time, by providing a background for the generation of their own research and formulate their own questions depending on their concerns and interests.

In fine, on the one hand, we considered the relevance of fabric analysis and the application of an archaeometric methodology to carry out a comprehensive assessment of the materials, techniques and manufacturing processes involved in pottery production as well as of the properties of the end products. However, it has also been emphasised that as archaeologists we must also interpret the features of the vessels according to the social context they participated in, considering issues that go beyond the materials, the natural environment or the subsistence basis and biological needs of the individuals.

This change in the analytical level, in which we go beyond the physical materiality to deal with the multiple social practices and intangible phenomena involved in the life cycle of ceramics, can be achieved by analysing the pottery in its historical context and by using of concepts such as agency, technological choice, habitus, technological tradition, social organisation of production, identity, transmission of information and knowledge, learning strategies, etc.

The combination of the huge corpus of data that can be obtained through archaeometric approaches along with these theoretical concepts and a deep contextual analysis provides an adequate framework for understanding the way pottery production is organised and clarifies the meaning and role of the pottery. Thus, ultimately, this methodology allows us to inquire about the organisation of a society itself. Moreover, this analytical strategy permits us to address some of the reasons why technological change takes place in pottery production and its relationship with 
the development of certain social dynamics in which the whole material culture is involved.

Nevertheless, before addressing such questions we must first be able to design analytical strategies meant to help identify the development of certain technological traditions through the study of both the material record and the archaeological context. In this sense, we have seen that the chaîne opératoire constitutes a suitable theoretical and methodological framework to study the features of the vessels, their properties and processes of physical change. This analytical framework focused on the features of the pottery and its properties allows tackling the level of specialisation of production, the potter's skills and the variability found in ceramic assemblages.

The features of the vessels also point to the existence of specific paste recipes, technological choices and pottery traditions whose characteristics are the consequence of the degree of interaction that took place between the individuals of a given social group. In short, the presence of these recipes as well as of a specific technological tradition demonstrates the existence of common practices that reinforce social ties and have significance in identity terms.

The application of multiple theoretical frameworks and viewpoints, among which we should include ethnoarchaeology and experimental archaeology, is crucial in order to carry out a more complex interpretation of material culture and its relation to social structure. However, it should not be forgotten that our assumptions and hypotheses are inevitably based on a comprehensive study of the archaeological record. Thus, the study of ancient technologies must necessarily consider material culture in order to understand the relation that takes place among materiality, praxis and social structure. In this sense, a precise and accurate characterisation of the vessels should be done in order to thoroughly address technology and its social dimension by means of the multiple technological choices involved in pottery production. In short, the study of pottery production should aim at characterising the different elements involved in the technological tradition as a whole.

Once the ceramic assemblages are characterized, it is possible to generate explanations regarding the way pottery production is socially organised and how ceramic technology interacts with the other technologies and social dynamics recorded through the analysis of other kinds of artefacts. This involves an inductive and deductive process that allows investigating how particular phenomena observed in the pottery are connected through time and space with diverse social dynamics and other kinds of structural phenomena. Thus, we should try to develop complex explanations involving different scales of analysis as well as to develop fractal-like models focused on the reproduction of the meanings attached to material culture at multiple levels and dimensions. To this end, it is absolutely necessary to relate first the features of the ceramics to their deposition and use contexts. Such an approach is crucial to understand whether specific ways of 
making pottery were related to archaeological sites associated with different contexts, activities and social dynamics. It is in these particular contexts of use and deposition of the vessels where, ultimately, the whole materiality actively interacted and made sense. 